\title{
Tracer Kinetic Model-Driven Registration for Dynamic Contrast Enhanced MRI Time Series
}

\author{
Giovanni A. Buonaccorsi ${ }^{1}$, Caleb Roberts ${ }^{1}$, Sue Cheung ${ }^{1}$, Yvonne Watson ${ }^{1}$, \\ Karen Davies ${ }^{1}$, Alan Jackson ${ }^{1}$, Gordon C. Jayson ${ }^{2}$, and Geoff J.M. Parker ${ }^{1}$ \\ 1 Imaging Science and Biomedical Engineering, \\ University of Manchester, Manchester, UK \\ giob@manchester.ac.uk \\ 2 Cancer Research UK Dept of Medical Oncology, \\ Christie Hospital, Manchester, UK
}

\begin{abstract}
Motion during time-series data acquisition causes modelfitting errors in quantitative dynamic contrast-enhanced (DCE) MRI studies. Motion correction techniques using conventional registration cost functions may produce biased results because they were not designed to deal with the time-varying information content due to contrast enhancement. We present a locally-controlled, 3D translational registration process driven by tracer kinetic modeling that successfully registers abdominal DCE-MRI data at high temporal resolution and compare this method to a similar approach based on registration to the time series mean image in data from 8 patients. When the registration is driven by an appropriate model, we find significant improvements in model-fitting. Also, model-driven registration influences parameter estimates and reduces repeat study variability in measurements of blood volume.
\end{abstract}

\section{Introduction}

The quantitative, model-based, analysis of contrast agent uptake kinetics in dynamic contrast enhanced MRI (DCE-MRI) at high temporal resolution allows us to estimate the magnitude and spatial distribution of physiological kinetic parameters such as $K^{\text {trans }}$ (the volume transfer coefficient of contrast agent between capillaries and the extravascular extracellular space - EES), $v_{e}$ (the volume of the EES per unit volume of tissue) and $v_{p}$ (the blood plasma volume per unit volume of tissue). These parameters provide useful information on microvascular status for studies of tumors and inflammatory conditions [123].

These parameters are usually estimated by deriving a contrast agent concentration time course and fitting a tracer kinetic model (e.g. 45]) within each voxel in a volume of interest (VOI). Model fitting errors arise because patient and physiological motion during data acquisition alters the voxel-to-tissue mapping. Conventional motion correction (e.g. registering each image volume in the time series to the time series mean [6]) has limited success because the passage of the contrast agent introduces new image features. The resulting time-varying information content is problematic even for inter-modal cost functions (e.g. mutual 
information [7]8, correlation ratio [9]), which are most effective with a consistent information content between the input and reference images.

Other investigators have registered pre-contrast to post-contrast images [10] and incorporated a simplified tracer kinetic model directly into a registration cost function [11, but it has not yet been practicable to register an entire DCEMRI time-series at high temporal resolution using a more detailed physiological model of enhancement in abdominal tumors. We propose a novel, tracer kinetic model-driven registration method that uses a computationally efficient, locallycontrolled, 3D translational registration focused on the tumor VOI.

\section{Method}

\subsection{Modelling}

We used the standard 4] and extended [5] Kety models as applied to DCEMRI data. The former assumes that the blood plasma volume, $v_{p}$, is small by comparison with the EES volume, $v_{e}$, and estimates $K^{\text {trans }}$ and $v_{e}$ only. This approximation fails in "rim-enhancing" tumors where angiogenesis may be pronounced [12, so in tumor studies it is usually more appropriate to use the extended Kety model, which includes a vascular term, $v_{p}$, and has the following integral form (note that the standard Kety model lacks the $v_{p} C_{p}(t)$ term):

$$
C_{t}(t)=v_{p} C_{p}(t)+K^{\text {trans }} \int_{t^{\prime}=0}^{t} C_{p}\left(t^{\prime}\right) \exp \left[\frac{-K^{\text {trans }}\left(t-t^{\prime}\right)}{v_{e}}\right] d t^{\prime}
$$

In Equation 1, $C_{t}(t)$ is the tracer concentration in the tissue (mMol), which varies with time, $t ; C_{p}(t)$ is the tracer concentration in blood plasma $(\mathrm{mMol})$ and $K^{\text {trans }}$ is expressed in $\min ^{-1}$.

Each model requires the contrast agent concentration in the arterial supply, $C_{p}(t)$. This arterial input function (AIF) must be measured or a functional form must be assumed. The standard Kety model [4] used a bi-exponential AIF based on contrast agent concentrations measured from blood samples taken at intervals after injection of Gd-DTPA in healthy volunteers. However, this simple AIF may cause significant errors in the estimation of tracer kinetic parameters [13] and where possible we derive a measured AIF from the DCE-MRI time series [14].

The models are expressed in terms of a contrast agent concentration time course, $C_{t}(t)$. We compute this from the MR signal intensities using the standard relations for a spoiled gradient echo acquisition [15. We estimate the parameters $K^{\text {trans }}, v_{e}$ and $v_{p}$ by fitting the above models to the measured data using a voxelby-voxel simplex optimization [16].

\subsection{Data Acquisition}

We acquired abdominal images on a Philips $1.5 \mathrm{~T}$ Intera MRI scanner using the whole body coil for transmission and reception. The conversion to $C_{t}(t)$ used a baseline $\mathrm{T}_{1}$ measurement comprising 3 axial spoiled gradient echo (Fast 
Field Echo) volumes with $2^{\circ}, 10^{\circ}$ and $20^{\circ}$ flip angles and 4 signal averages. The dynamic series comprised 75 consecutively-acquired axial volumes with a $20^{\circ}$ flip angle, 1 average and a $4.97 \mathrm{~s}$ temporal resolution. All studies used 25 slices, $375 \times 375 \mathrm{~mm}^{2}$ field of view, $128 \times 128$ matrix, $4.0 \mathrm{~ms} T R$, and $0.82 \mathrm{~ms}$ $T E$. Slice thickness was 4 or $8 \mathrm{~mm}$. Tumor VOIs were defined independently of the registration study by an expert health professional, based on co-localized $\mathrm{T}_{2}$-weighted and $\mathrm{T}_{1}$-weighted pre- and post-contrast image volumes.

Motion was graded (also independently of the registration study) on a 4 point scale. For registration, we selected 10 data sets from 8 patients with abdominal tumors who scored "moderate" or "severe" on this scale. In 5 patients we were also able to assess the reproducibility of fitted parameters via scan-rescan tests.

\subsection{Registration}

We assumed the tumors to be small relative to the abdomen, and to be more rigid than their surrounding tissues [17. This allowed us to use a 3D translational registration procedure that calculated the registration cost function only within the tumor VOI but applied the resulting translation to the whole image volume. All registrations used FLIRT [18] (from the FSL package http://www.fmrib.ox. ac.uk/fsl) with the following iterative scheme.

Step 1: Fit the chosen model to the original, motion-corrupted, time series data within the tumor VOI to obtain initial estimates of $K^{\text {trans }}, v_{e}$ and $v_{p}$.

Step 2: Output 3D maps (volumes) of the signal intensities generated by the model fit (back-converted from the modelled concentration values) for each time point in the dynamic series. These 3D maps are synthetic image volumes for which the intensity in each voxel is the output of a model fit that depends on the whole original time series. The synthetic maps therefore display no motion from time point to time point and can be used as reference volumes to drive the registration of the original time point image volumes.

Step 3: Perform a 3D translational registration using a standard FLIRT cost function, matching each original time point volume to its corresponding synthetic reference volume. To achieve local control, we calculate the registration cost function only within the tumor VOI. For robustness, we reject registrations where the translation magnitude (extracted from the translation matrix output by FLIRT) exceeds visually-defined extremes of motion for the given data set.

Step 4: Re-fit the chosen model to the registered time series.

Step 5: Repeat Steps 2-4 until a minimum is found in the median model fit sum of squared errors within the tumor VOI, at each stage fitting the model to the "last-registered" time series to generate new synthetic reference images then registering the "last-registered" time series to the new synthetic images.

We tested the registration using synthetic reference images from the standard Kety model with the bi-exponential AIF (labelled STD-EXP) and the extended Kety model with a measured AIF (EXT-MSD). For comparison with a more standard registration procedure, we also used the time-series mean image as the synthetic reference (we may view the time series mean as the simplest model of time-varying signal). In this case the EXT-MSD model was applied to the data 
after each time-series mean registration loop and the SSE calculated to compare results with the model-driven registration.

\section{Results}

Figure 1 compares the sum of squared errors (SSE) on the model fits after modeldriven registration and registration to the time series mean. As localized failures in the voxel-by-voxel fitting process may give outliers in the individual voxel SSE, for robustness we quote the median SSE over the tumor VOI. Registration significantly reduced the SSE (i.e. reduced model fitting errors) for 8 of the 10 data sets in each group. The SSEs were generally lower for registration to the time series mean than for model-driven registration, implying that the time series mean image was a more effective registration target. However, this global statistic hides some important details that we will discuss later. Note that the magnitudes of the recovered translations were always less than $15 \mathrm{~mm}$ in the through-plane (i.e. superior-inferior) direction and less than $8 \mathrm{~mm}$ in the inplane directions, which is reasonable for these data sets.

Figure 2 quantifies the changes from the pre-registration median tumor VOI estimates of $K^{\text {trans }}, v_{e}$ and $v_{p}$ after EXT-MSD model-driven registration and registration to the time-series mean. Model-driven registration resulted in greater changes for all three parameters.

Figure 3 plots the model fits and data for a small VOI within the enhancing rim of a tumor in one data set, before and after registration. The data show the expected features of a "first pass" peak in contrast agent concentration, followed by a prolonged washout. In each case the post-registration data (circles) cluster more tightly around the fitted curve than the pre-registration data (crosses), consistent with a reduced SSE. However, STD-EXP model-driven registration and registration to the time series mean distorted the data behavior around the

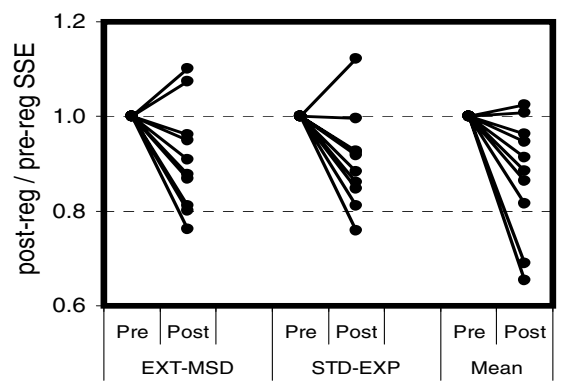

Fig. 1. Post-registration SSE relative to its pre-registration value for model-driven registration and for registration to the time series mean image - a downward slope indicates reduced SSE

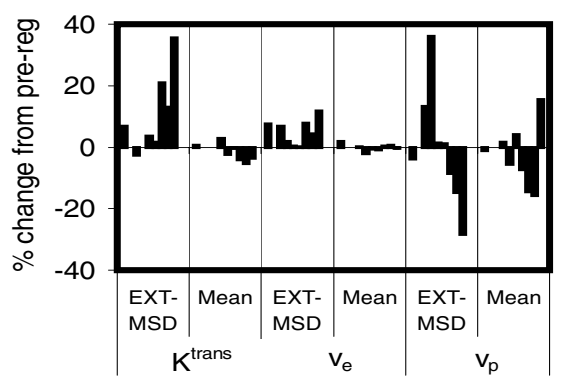

Fig. 2. Changes in the median tumor VOI estimates of the model parametes after model-driven and time series mean image registration, expressed as a percentage change from the pre-registration estimates 

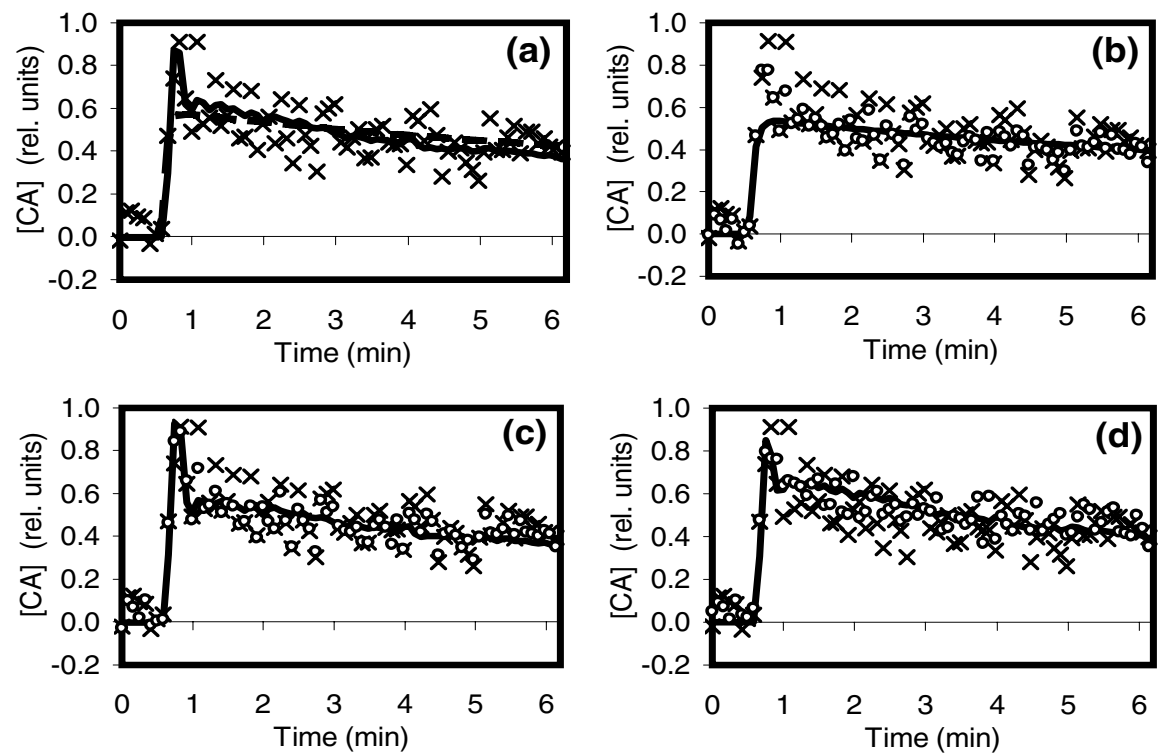

Fig. 3. Model fit curves with data before and after registration. The lines are the fitted models, the crosses pre- and the circles post-registration data. (a) Pre-registration fits for EXT-MSD (solid) and STD-EXP (dashed). Post-registration fits for (b) STDEXP and (c) EXT-MSD model-driven registration and for (d) an EXT-MSD fit after registration to the time-series mean.

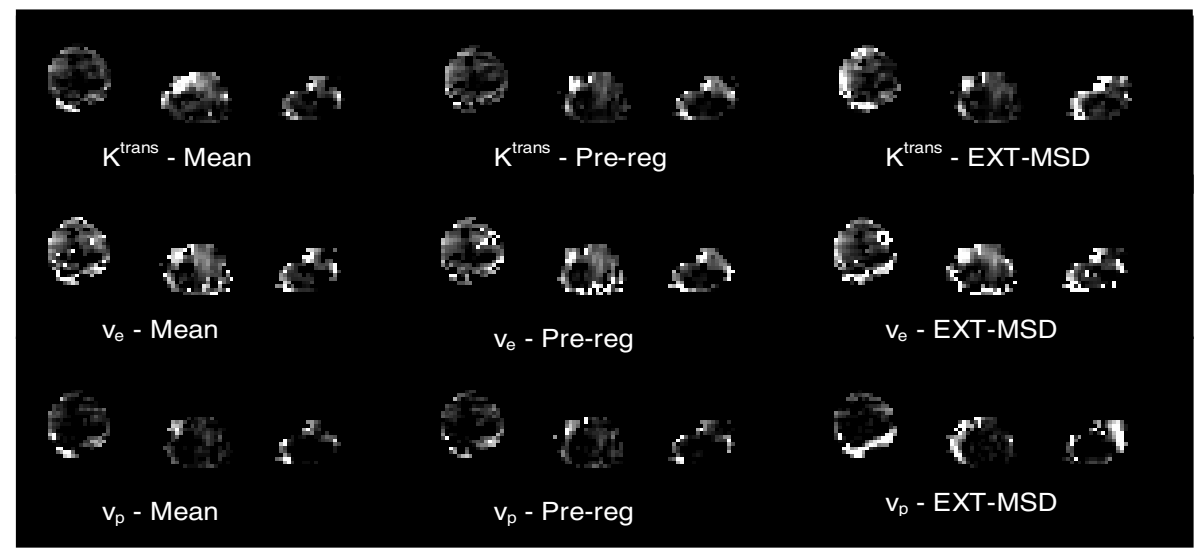

Fig. 4. Maps of the estimated parameters before registration (center column) and after registration to the time series mean (left) and EXT-MSD model-driven registration (right). In each set, the left-hand map is the axial view, the center map the sagittal view and the right-hand map the coronal view. Gray scale values are consistent for each parameter and lighter shading corresponds to higher parameter values. Gray scale ranges: $K^{\text {trans }} 0-0.4 \mathrm{~min}^{-1} ; v_{e} 0-0.8 ; v_{p} 0-0.2$. 
first-pass peak, while EXT-MSD model-driven registration did not (we discuss this point more fully later in the paper).

Figure 4 shows the effect of registration on the spatial localization of parameter estimates. There are clear differences when comparing post-registration parameter maps to pre-registration maps for EXT-MSD model-driven registration - in particular, the maps of $v_{p}$ and $K^{\text {trans }}$ show a more clearly-defined enhancing rim with higher parameter values. However, the differences after registration to the time series mean are less evident. These observations are compatible with the post-registration changes in the median VOI parameter values, which were always of greater magnitude after model-driven registration (Figure 2).

For 5 patients, we were able to perform scan-rescan tests to establish whether registration could reduce inter-visit variability in parameter estimates. We found only one significant reduction after EXT-MSD model-driven registration (in $v_{p}$, $P<0.05$ for a paired one-tailed t-test on the absolute percent changes in parameter estimates between 2 visits, before and after registration) and no significant reductions after time series mean registration.

\section{Discussion}

Conventional motion correction using standard registration cost functions does not cope well with features that appear and disappear between images, as happens when using a contrast agent. Investigators have observed biased output when using mutual information in a non-rigid registration algorithm for pre- vs. post-contrast MR images of focal breast lesions [19], and addressed this by adding a constraint to the cost function to penalize the artefactual deformations arising in the enhancing region of post-contrast images [20. Another group successfully incorporated a simplified analytical form of a kinetic model into a non-rigid registration cost function [11.

Neither of these approaches can be directly applied when fitting more comprehensive kinetic models to DCE-MRI time series data from abdominal tumors at high temporal resolution. We have therefore developed a locally-controlled, 3D translational registration method that employs synthetic reference images generated from tracer kinetic model fits or from the time series mean and evaluated its performance using the standard and extended Kety models. We significantly reduced the SSE for $8 / 10$ data sets in each group, indicating that in general registration to a synthetic reference image reduces motion corruption in the time series. Registration to the time series mean gave the greatest SSE reduction, implying that the mean image provides the best reference, but the SSE is a global statistic that hides some important details that contradict this conclusion.

We can see these details by looking at the data and model fits for a small VOI within the enhancing rim of a tumor (Figure 3). Both registration to the time series mean and standard Kety model-driven registration distorted the time-series data in a manner that is inconsistent with the expected and experimentally-observed behavior of DCE-MRI data. Registration to the time 
series mean reduced the height of the first-pass peak in contrast agent concentration and gave raised concentrations during the washout phase, showing that the averaging process reduces the scatter in the data at the expense of important genuine structure. Standard Kety model-driven registration also reduced the height of the first-pass peak — it is significant that this model does not account for the blood plasma pool and so cannot accurately model this peak. Such distortions were not observed with the extended Kety model, which does account for the blood plasma pool. Therefore, if kinetic model-driven registration is to be successful, we must use a model that adequately characterizes the time course.

Extended Kety model-driven registration gave greater changes in median tumor VOI estimates of $K^{\text {trans }}, v_{e}$ and $v_{p}$ than registration to the time-series mean, but the interpretation of these changes is unclear, partly because these are median values from a heterogeneous volume. In the 3D parameter maps, extended Kety model-driven registration gave higher localized values of $K^{\text {trans }}$ and $v_{p}$, with a more defined enhancing rim pattern consistent with typical tumor structure. However, without ground truth data we cannot determine whether these changes are "correct" (future studies will address this problem using synthetic images for which ground truth is available). It is nevertheless significant that $K^{\text {trans }}$ and $v_{p}$ depend strongly on the first-pass peak, which we have seen to be distorted by time series mean registration but maintained by extended Kety model-driven registration.

Only extended Kety model-driven registration gave a statistically significant reduction in the variability of parameter estimates across 2 DCE-MRI studies in 5 patients, and only with $v_{p}$. Nevertheless, this suggests that modeldriven registration may reduce variability and thereby improve the sensitivity of quantitative DCE-MRI to genuine parameter changes - a factor of significant interest to clinical trials of novel anti-vascular and anti-angiogenic drugs (see e.g. [3]).

It is encouraging to have obtained such positive results from a simplified registration procedure. Our 3D translational registration is clearly not sufficient to fully recover tumor motion, which will include rotations and shape changes, requiring a non-linear or warping registration, and we hope to address this in future work. Despite its limitations, our registration process has reduced the adverse effects of motion in quantitative DCE-MRI studies, and shown that the kinetic model must be sufficiently detailed to avoid distorting the time-course data. Our implementation is fast enough for routine use (c. 2 hours to register 75 time point image volumes on a standard PC).

Based on the evidence in this paper, we propose that kinetic model-driven registration is a useful additional tool for the quantitative analysis of DCE-MRI data that addresses the problem of changing image information content (due to contrast enhancement) that can confound conventional registration methods.

Acknowledgements. We would like to acknowledge the financial support received from Cancer Research UK. 


\section{References}

1. Tofts, P., Brix, G., Buckley, D., et al.: Estimating kinetic parameters from dynamic contrast-enhanced $\mathrm{T}_{1}$-weighted MRI of a diffusable tracer: standardized quantities and symbols. J. Magn. Reson. Imag. 10 (1999) 223-32

2. Parker, G., Padhani, A.: $T_{1}$-weighted dynamic contrast-enhanced MRI. In Tofts, PS, ed.: Quantitative MRI of the Brain. 1st edn. Wiley, New York (2003)

3. Jackson, A and Buckley, DL and Parker, GJM, ed.: Dynamic contrast-enhanced magnetic resonance imaging in oncology. 1st edn. Springer, Berlin (2005)

4. Tofts, P., Kermode, A.: Measurement of the blood-brain barrier permeability and leakage space using dynamic MR imaging. I. Fundamental concepts. Magn. Reson. Med. 17 (1991) 357-67

5. Tofts, P.: Modeling tracer kinetics in dynamic Gd-DTPA MR imaging. J. Magn. Reson. Imag. 7 (1997) 91-101

6. Jenkinson, M., Bannister, P., Brady, J., et al.: Improved optimisation for the robust and accurate linear registration and motion correction of brain images. Neuroimage 17 (2002) 825-41

7. Wells III, W., Viola, P., Atsumi, H., et al.: Multi-modal volume registration by maximisation of mutual information. Med. Imag. Anal. 1 (1997) 35-51

8. Maes, F., Collignon, D., Vandermeulen, D., et al.: Multimodality image registration by maximisation of mutual information. IEEE Trans. Med. Imag. 16 (1997) 187-98

9. Roche, A., Malandain, G., Pennec, X., et al.: The correlation ratio as a new similarity measure for multimodal image registration. Lect. Notes Comput. Sc. 1496 (1998) 1115-24

10. Rueckert, D., Sonoda, L., Hayes, C., et al.: Nonrigid registration using free-form deformations: application to breast MR images. IEEE Trans. Med. Imag. 19 (1999) $712-21$

11. Hayton, P., Brady, M., Tarassenko, L., et al.: Analysis of dynamic MR breast images using a model of contrast enhancement. Med. Imag. Anal. 1 (1997) 207-24

12. Folkman, J.: Role of angiogenesis in tumor growth and metastasis. Semin. Oncol. 29 (2002) 15-18

13. Parker, G., Tanner, S., Leach, M.: Pitfalls in the measurement of tissue permeability over short time-scales using multi-compartment models with a low temporal resolution blood input function. Proc. ISMRM 4 (1996) 1582

14. Parker, G., Jackson, A., Waterton, J., et al.: Automated arterial input function extraction for $\mathrm{T}_{1}$-weighted DCE-MRI. Proc. ISMRM 11 (2003) 1264

15. Haase, A.: Snapshot FLASH MRI. Application to $\mathrm{T}_{1}, \mathrm{~T}_{2}$ and chemical shift imaging. Magn. Reson. Med. 13 (1990) 77-89

16. Press, WH and Teukolsky, SA and Veterling, WT and et al.: Numerical Recipes in C. 2nd edn. Cambridge University Press, Cambridge, UK (1992)

17. Sarvazyan, A., Goukassian, D., Maevsky, E., et al.: Elastic imaging as a new modality of medical imaging for cancer detection. Proc. Int. Workshop on Interaction of Ultrasound with Biological Media, (1994) 69-81

18. Jenkinson, M., Smith, S.: A global optimisation method for robust affine registration of brain images. Med. Imag. Anal. 5 (2001) 143-56

19. Tanner, C., Schnabel, J., Chung, D., et al.: Volume and shape preservation of enhancing lesions when applying non-rigid registration to a time series of contrast enhancing breast images. Lect. Notes Comput. Sc. 1935 (2000) 327-37

20. Tanner, C., Schnabel, J., Degenhard, A., et al.: Validation of volume-preserving non-rigid registration: application to contrast-enhanced MR mammography. Lect. Notes Comput. Sc. 2488 (2002) 307-14 